Gynäkologe 2021 · 54:468-470

https://doi.org/10.1007/s00129-021-04822-4

Angenommen: 20. Mai 2021

๑) Springer Medizin Verlag GmbH, ein Teil von Springer Nature 2021

In einer Zeit provokant demonstrierter Ganzheitlichkeit und Natürlichkeit erscheint der Terminus „künstliche Intelligenz" geradezu als Anachronismus. Allerdings wissen Personen aus dem medizinischen Milieu sehr wohl, dass ihre Aufgabe zu einem erheblichen Teil darin besteht, natürlichen Problemen mit u. U. grausamen Konsequenzen entgegenzustehen - und dies nicht nur auf natürliche Weise. Entsprechend müsste es uns aus dem Medizinsektor in den Ohren klingeln, wenn wir besser, präziser, schneller, effektiver diagnostizieren und handeln könnten mithilfe nützlicher intelligenter Algorithmen zur Bewältigung komplexer Probleme. So erleichtern automatisierte Bildanalysen bisher überwiegend die radiologische, aber auch die zytologische Arbeit. Die Spracherkennung beim Diktat von Befunden ist in den meisten Kliniken schon Alltag geworden, und viele neue Anwendungen im Alltag stehen bereits in Sichtweite.

Oder bekommen wir es mit der Angst zu tun, wenn uns der Gedanke der Ersetzbarkeit durch künstliche Intelligenz bemannt angesichts diagnostischer Kombinatorik, die jenseits der Möglichkeiten von Einzelpersonen oder Gruppen liegt, die mit extremer Geschwindigkeit einsetzbar ist und die - und hier könnte es kritisch werden - keinen Arztvorbehalt kennt?

Unbegrenzte, allerdings auch ungefilterte Informationen können den Laien in die Situation einer punktuellen Überinformation versetzen, die er - mangels adäquater Filter - zu bewältigen nicht in der Lage ist. Dies auszubalancieren

R. Kreienberg ist vor der Veröffentlichung dieses Beitrags leider verstorben.

\author{
Rolf Kreienberg ${ }^{1}$ ' Wolfgang Janni ${ }^{2} \cdot$ Klaus Vetter $^{3}$ \\ 'Mainz, Deutschland \\ ${ }^{2}$ Frauenheilkunde und Geburtshilfe, Universitätsklinikum Ulm, Ulm, Deutschland \\ ${ }^{3}$ Berlin, Deutschland
}

\title{
Künstliche Intelligenz (KI)
}

benötigen wir Kenntnisse jenseits rein medizinischen Wissens.

Der homo contemplator wird durch den Besitz von Bewegungs-SteigerungsMitteln zum homo navigator auf dem Daten-Ozean (Sloterdijk).

Worum geht es angesichts unbegrenzter Assoziationen und ausgelöster Vorstellungen durch den Begriff künstliche Intelligenz?

Ging es anfangs um Unterstützung des Erkennens und Denkens durch Rechner, Computer und Algorithmen, ist der Alltag heute durchsetzt mit nicht nachgefragten Informationen via Handy, Uhr oder Wearables, die permanent Daten sammeln und verarbeiten können. Mit Großrechnern verbunden sind sie in der Lage, Bewegungsmuster zu analysieren auf Hinweise für Erkrankungen, wie M. Parkinson oder COVID(„,coronavirus disease")-19.

Intelligenz umschreibt die Fähigkeit oder kognitive Leistung, sich in neuen Situationen zurechtzufinden und Aufgaben durch Denken zu lösen. Moderne Ansätze unterteilen sie gerne in die mathematische, sprachliche, technische, musische, emotionale oder soziale Intelligenz.

Von künstlicher Intelligenz spricht man dann, wenn das Ergebnis eines Algorithmus-gesteuerten Vorgangs von einem Menschen-gesteuerten im Prinzip nicht zu unterscheiden ist. In praxi soll sie es Maschinen im weitesten Sinn ermöglichen, aus Erfahrungen zu lernen, sich an neue Eingaben anzupassen und menschenähnliche Aufgaben auszuführen. In den letzten Jahren wurden Unterkategorien etabliert, erst „machine learning“, später „deep learning“ (• Abb. 1).

Künstliche Intelligenz bedeutet den Einsatz von Computern zur Modellie- rung intelligenten Verhaltens mit minimalem menschlichen Beitrag, d. h. Nachahmung menschlichen Verhaltens: fühlen, denken, handeln und anpassen, nicht aber: entscheiden oder bewerten.

Machine Learning bedingt, dass Algorithmen auf Basis zunehmender Daten, vulgo Erfahrung, optimiert werden. So ergeben sich beim „supervised learning “ - auf früheren, schon bekannten Erfahrungen aufbauend - Klassifizierungsund Vorhersagealgorithmen.

Beim „unsupervised learning“ geht es um das Finden bisher nicht bekannter Muster; und beim „reinforcement learning " schließlich werden positive Ergebnisse durch Verstärkung gefördert.

\section{》) Künstliche Intelligenz kann den Menschen als Maß der Dinge nicht ersetzen}

Deep Learning ermöglicht das Lernen aus großen Datenmengen mittels neuronaler Netze. Die Tiefe entspricht den Knotenpunktschichten. Das Netzwerk kann sehr komplex werden und dadurch ähnlich einer Black Box kaum mehr durchschaut und kontrolliert werden. Dabei geht es um Mustererkennung und Prognosemodelle. So hat sich Deep Learning in den letzten Jahren als führendes Element der künstlichen Intelligenz entwickelt, das uns die Möglichkeit bietet, aus Rohdaten komplexe Funktionen mit beispielloser Genauigkeit und in noch nie dagewesenem Umfang zu analysieren.

Künstliche Intelligenz kann den Menschen als Maß der Dinge nicht ersetzen. Es handelt sich bei künstlicher Intelligenz um Versuche der Nachahmung menschlichen Verhaltens inklusive fühlen, denken, handeln, anpassen etc. Aber sele- 


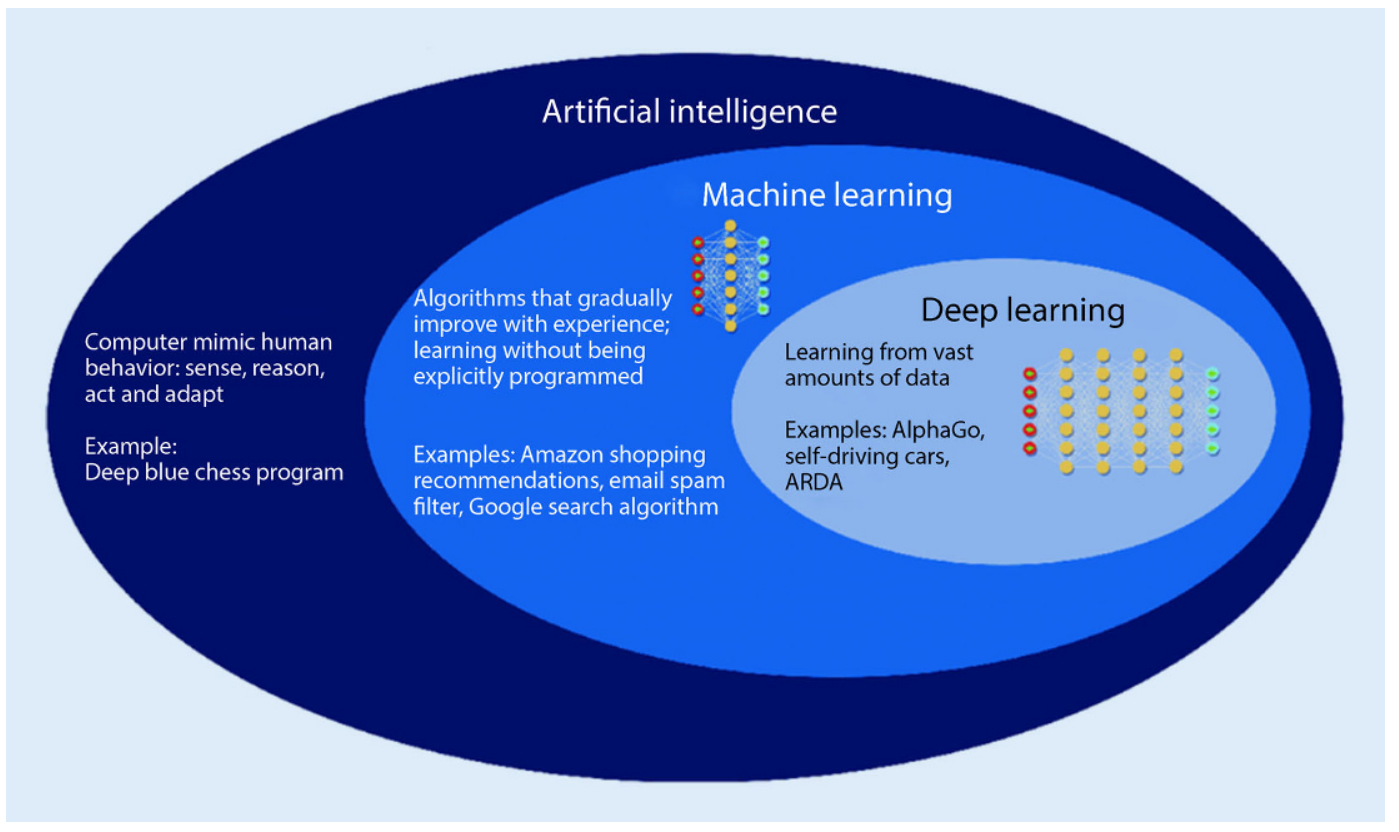

Abb. $1 \triangleleft$ Beziehung zwischen künstlicher Intelligenz, "machine learning" und "deep learning". $A R$ $D A$ "automated retinal disease assessment". (Mod. nach [1]. Licenced under CCBY 4.0: https:// creativecommons.org/ licenses/by/4.0/)

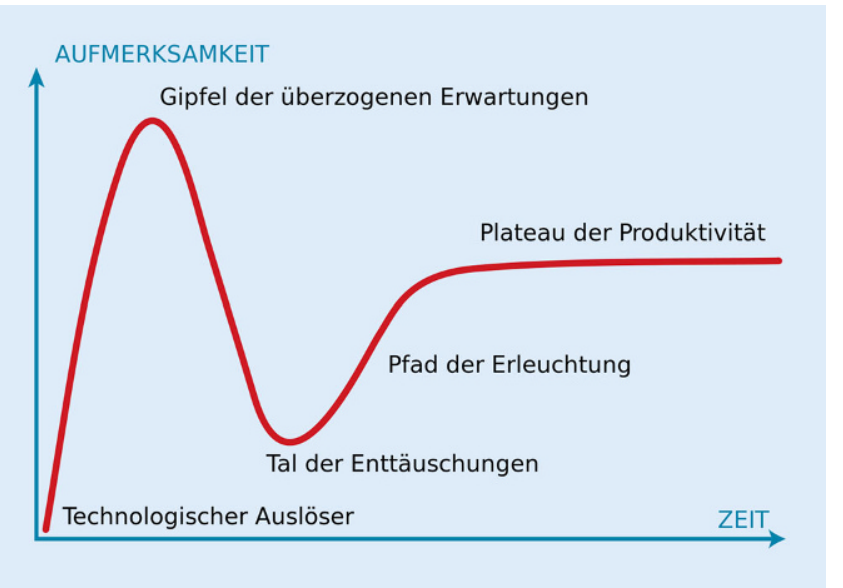

Abb. $2<\mathrm{Hy}$ pe-Zyklus nach Gartner. (Aus [2]. Licenced under CCBY 3.0: https:// creativecommons.org/ licenses/by-sa/3.0/ deed.de) gieren, bewerten und entscheiden bleibt der künstlichen Intelligenz vorenthalten, solange man dies nicht bewusst einem Algorithmus überantwortet. Levine titelte 2017 „Artificial intelligence has uses in medicine, but will never replace doctors' intuition" [3].

In diesem Heft stellen wir sehr unterschiedliche Aspekte der künstlichen Intelligenz vor. Den Herausgebern war es dabei wichtig, dieses Heft mehr der Bedeutung und den Perspektiven künstlicher Intelligenz $\mathrm{zu}$ widmen als ihrem Einfluss auf detaillierte medizinische Fragestellungen.

Walter Ch. Zimmerli führt mit „Künstliche Intelligenz - ein Mythos des 21. Jahrhunderts?" in die aktuelle philosophische Situation auf Basis der Entste- hungsgeschichte ein und weist zentral auf die Dynamik der Rezeption mittels des Gartner-Hype-Zyklus hin (• Abb. 2).

Wäre einmal der Zustand erreicht, dass die geschaffene („künstliche“) Intelligenz alle menschlichen („natürlichen“) Intelligenzleistungen überstiege, gäbe es für diese auch keine Möglichkeit mehr, die dadurch eröffneten Zukunftsoptionen vorherzusagen.

Auf jeden Fall wird aber die Philosophie, zumal die Erkenntnis- und Wissenschaftstheorie, über die Bücher gehen und die Einsicht ernst nehmen müssen, dass Wissen nicht nur von Menschen, sondern auch von Mensch-Maschine-Tandems generiert werden kann. Das heißt jedoch nicht, dass künstliche
Intelligenz diejenige der Menschen ersetzen oder gar überflüssig machen würde.

Daniel Sonntag geht in seinem Beitrag „Künstliche Intelligenz in der Medizin - Holzweg oder Heilsversprechen?" auf praktische Berührungspunkte mit der Medizin ein und weist dezidiert auf die Grenzen der künstlichen Intelligenz in der Medizin hin. Entscheidend für die erfolgreiche Anwendung künstlicher Intelligenz seien der Zugang zu qualitativ hochwertigen Daten und die Integration in komplexe medizinische Dienstleistungen im klinischen und nichtklinischem Umfeld. Und insbesondere sagt Sonntag, dass es den Künstlichen-IntelligenzSystemen bei Entscheidungen in lebensbedrohlichen Situationen an der nötigen Robustheit fehle.

\section{1) Wissen kann nicht nur von Menschen, sondern auch von Mensch-Maschine-Tandems generiert werden}

Oliver Kagan und Markus Hoopmann beschäftigen sich mit dem Einfluss künstlicher Intelligenz auf die Pränataldiagnostik. Hier wird deutlich, dass es sich bei einem Schachcomputer mit $10^{47}$ Möglichkeiten um ein überschaubares Problem handelt, das beim Go-Spiel mit $10^{170}$ Möglichkeiten schon erheblich größer ist, 
Fachnachrichten

aber durch Fragen der Biologie bei weitem getoppt wird. In ihrem Beitrag „Bestehende und zukünftige Assistenzsysteme in der pränatalmedizinischen Bildgebung" zeigen sie, in welch dynamischer Entwicklung sich die Pränatalmedizin insbesondere durch lernende Assistenzsysteme bei der Sonographie befindet. „Die Einführung von Assistenzsystemen, die auf künstlicher Intelligenz beruhen, haben das Potenzial, die sonographische Untersuchung bezüglich Wirksamkeit („efficacy“), Effektivität („efficiency") und Qualität grundlegend zu verändern."

Kurt J. G. Schmailzlbehandelt schließlich die künstliche Intelligenz in Zusammenhang mit der Kunst der Medizin. Diese bleibe - so unsere Hoffnung Menschenwerk. Das könne sie aber auch schon unter aktuellen Randbedingungen nur dann, wenn wir unsere ärztlichen Aufgaben entsprechend sehen und definieren. Künstliche Intelligenz habe das Potenzial, uns Menschen so gut zu durchschauen und zu „verstehen“, dass sie humane Zwecke usurpieren und uns kontrollieren könnte. Insofern sei unsere Intelligenz gefordert, die neue, die künstliche, einzuhegen. Ob wir mit den Risiken, welche die künstliche Intelligenz mit sich bringt, fertig werden und überhaupt eine Chance haben, die mit ihr aufgeworfenen Probleme zu lösen, hänge mehr vom ethischen und politischen als vom fachmedizinischen Diskurs ab. Aber dazu müssten wir künstliche Intelligenz zu einem Schlüsselthema machen.

Das genau haben wir hiermit getan, auch im Hinblick auf Artikel 3 Künstliche Intelligenz aus Ferdinand von Schirachs Vorschlag für eine Erweiterung der EU-Menschenrechtscharta [4]: „Jeder Mensch hat das Recht, dass ihn belastende Algorithmen transparent, überprüfbar und fair sind. Wesentliche Entscheidungen muss ein Mensch treffen".

Rolf Kreienberg $\dagger$

Wolfgang Janni

Klaus Vetter

\section{Korrespondenzadresse}

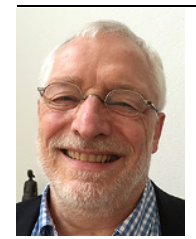

Prof. Dr. Klaus Vetter

Friedrichshaller Str. 7c, 14199 Berlin, Deutschland kvetter@ngi.de

Interessenkonflikt. R. Kreienberg, W. Janni und K. Vetter geben an, dass kein Interessenkonflikt besteht.

\section{Literatur}

1. Drukker L, Noble JA, Papageorghiou AT (2020) Introduction to artificial intelligence in ultrasound imaging in obstetrics and gynecology. Ultrasound Obstet Gynecol. https://doi.org/10.1002/uog. 22122

2. https://de.wikipedia.org/wiki/Datei:Gartner_ Hype_Zyklus.svg.Zugegriffen: 14. Mai 2021

3. Levine BA (2017) Al has brains - but it's no MD. Contemporary OB/GYN.62(4)

4. von Schirach F (2021) Jeder Mensch. München: Luchterhand Verlag

\section{Schutz vor sexuell übertrag- baren Infektionen}

BZgA-Initiative LIEBESLEBEN startet Plakatkampange »Hautnah«

Mit aufmerksamkeitsstarken Plakatmotiven der Initiative LIEBESLEBEN, die viel Haut zeigen, informiert die Bundeszentrale für gesundheitliche Aufklärung (BZgA) ab Juni bundesweit zum Schutz vor sexuell übertragbaren Infektionen (STI). Die »Hautnah«-Kampagne motiviert sexuell aktive Menschen, sich selbst zu schützen und auf den Schutz von Partnerinnen und Partnern zu achten. Gleichzeitig empfiehlt sie, sich bei Symptomen auf eine sexuell übertragbare Infektion testen zu lassen.

In der ärztlichen Praxis besteht nach wie vor Beratungsbedarf zu den - neben HIV/AIDS - weiteren sexuell übertragbaren Infektionen. Die BZgAInitiative LIEBESLEBEN unterstützt deshalb auch Ärztinnen und Ärzte in ihrer STIPräventionsarbeit. Die GeSiD-Studie zu Gesundheit und Sexualität in Deutschland ergab, dass nur 21 Prozent der Männer und 31 Prozent der Frauen mit einem Arzt oder einer Ärztin über sexuell übertragbare Infektionen gesprochen haben. Ein verdächtiges Symptom, mit dem Patientin oder Patient in die Praxis kommen, kann der erste und zugleich späte Anlass sein, über STI zu informieren. Dabei kann eine frühzeitige arztseitige Aufklärung über STI die möglichen Maßnahmen zur Prävention, beispielsweise mit Kondomen, aufzeigen und Infektionen samt möglicher Spätfolgen vorbeugen.

Weiterführende Informationen und Materialien für Fachkräfte bietet die BZgA unter:

www.liebesleben.de/fachkraefte

Quelle: Presseinformation LIEBESLEBEN, 01. Juni 2021 